Anuario Latinoamericano Ciencias Políticas

y Relaciones Internacionales

vol. 7, 2019

pp. 131-151

DOI: 10.17951/al.2019.7.131-151

\section{La gobernanza de la seguridad alimentaria en América Latina: desde la cooperación Norte-Sur hacia la cooperación Sur-Sur}

\author{
Food Security Governance in Latin America: \\ from North-South to South-South Cooperation
}

\author{
Katarzyna Marzęda-Młynarska* \\ FACULTAD DE CIENCIAS POLÍTICAS Y PERIODISMO \\ UNIVERSIDAD MARIA CURIE-SKŁODOWSKA \\ LUBLIN, POLONIA \\ $\triangle$ katarzyna.marzeda@poczta.umcs.lublin.pl \\ https://orcid.org/0000-0002-4608-7290
}

\title{
RESUMEN
}

Combatir el hambre y la desnutrición y garantizar la seguridad alimentaria son prioridades globales de acción, establecidas en la Agenda 2030, en el Objetivo 2: Hambre Cero. Su implementación requiere una acción compleja y coordinada a nivel nacional e internacional y el desarrollo de un modelo efectivo de gobernanza de la seguridad alimentaria en el futuro. América Latina es un ejemplo interesante de la regionalización de la gobernanza de la seguridad alimentaria. El estudio tiene como objetivo analizar el modelo latinoamericano con especial énfasis en el papel de las organizaciones regionales y demostrar que el modelo regional de gobernanza de la seguridad alimentaria se basa en el mecanismo de cooperación Sur-Sur, que promueve la cooperación y las acciones conjuntas de los países en desarrollo. El análisis se basa en dos supuestos: primero, debido a la naturaleza multidimensional de la seguridad alimentaria, está creciendo la importancia de la cooperación y las soluciones multilaterales; segundo, ante los nuevos desafíos, el modelo de la cooperación Norte-Sur, que domina el desarrollo internacional, se vuelve insuficiente y tiene que complementarse con otros arreglos. Como resultado, la cooperación Sur-Sur e intrarregional está ganando importancia en América Latina.

* Doctora en Ciencias Políticas. Profesora adjunta en la Facultad de Ciencias Políticas de la Universidad Maria Curie-Skłodowska en Lublin. Sus intereses de investigación incluyen temas de gestión global, seguridad alimentaria y economía política internacional ampliamente entendida. Autora de publicaciones sobre relaciones internacionales contemporáneas, con especial énfasis en seguridad alimentaria, gestión global, corporaciones transnacionales, procesos de globalización y problemas globales. 
Dossier América Latina: continuidad y cambio en el escenario regional
PALABRAS CLAVE: gobernanza regional, seguridad alimentaria, gobernanza de la seguridad alimentaria, América Latina, cooperación Sur-Sur, Objetivos de Desarrollo Sostenible.

\begin{abstract}
Combating hunger and malnutrition and ensuring food security are global priorities for action, set out in Agenda 2030, under Goal 2: Zero Hunger. Its implementation requires complex and coordinated action at both national and international levels and the development of an effective model of food security governance in the future. Latin America is an interesting example of the regionalization of food security governance. The study aims to analyze the Latin American model with particular emphasis on the role of regional organizations, and to demonstrate that the regional food security governance model is based on the South-South cooperation mechanism, which promotes cooperation and joint actions of developing countries. The analysis is based on two assumptions: first, due to the multidimensional nature of food security, the importance of cooperation and multilateral solutions is growing; second, the NorthSouth model of cooperation, which dominates international development, becomes insufficient in the face of new challenges and is supplemented by other arrangements. As a result, the South-South and intra-regional cooperation is gaining in importance in Latin America.
\end{abstract}

KEYWORDS: regional governance, food security, food security governance, Latin America, South-South cooperation, Sustainable Development Goals.

\section{Introducción}

La comida es la necesidad existencial del hombre, de cuya satisfacción depende no solo la supervivencia de la humanidad en el sentido biológico, sino también su futuro desarrollo. No sin razón muchas civilizaciones antiguas surgieron en lugares que ofrecían condiciones favorables para la producción de los alimentos, como el acceso al agua o suelos fértiles, y colapsaron cuando estas condiciones sufrieron cambios drásticos. A lo largo de los años, el hombre aprendió a usar las condiciones ambientales y a administrar los recursos para garantizar el acceso a los alimentos, sin embargo, sus esfuerzos a la larga resultaron ser insuficientes. Independientemente de la época histórica y el nivel del desarrollo civilizacional, el hambre y la desnutrición no se han eliminado y una parte importante de la humanidad sigue amenazada por ellos.

También hoy en día este problema sigue teniendo una importancia vital. Ante las crecientes tendencias negativas, que incluyen el crecimiento de la población mundial, la urbanización progresiva y los cambios climáticos, incrementan preocupaciones sobre la posibilidad de garantizar la seguridad alimentaria a escala mundial. Las acompañan pronósticos alarmantes que indican una creciente competencia por los recursos necesarios desde el punto 
de vista de la producción de alimentos (Koba 2014). Por esta razón, la lucha contra el hambre es un área prioritaria de las actividades de la comunidad internacional emprendidas como parte de los Objetivos de Desarrollo del Milenio (ODM) y, actualmente, los Objetivos de Desarrollo Sostenible (ODS).

La complejidad del problema se refleja en el concepto de seguridad alimentaria, creado y popularizado por la Organización de las Naciones Unidas para la Alimentación y la Agricultura (FAO), dentro del cual se han distinguido cuatro dimensiones: física, económica, de salud y de tiempo ${ }^{1}$; y cinco niveles: individual, de hogar, nacional, regional y global (FAO 1996). La mera provisión de la seguridad alimentaria, es decir, de acuerdo con la definición: "[proporcionando - KMM] a cada persona en todo momento el acceso físico, social y económico a alimentos seguros y nutricionalmente adecuados, suficientes en términos de necesidades y preferencias nutricionales, y asegurando una vida activa y saludable" (FAO 2009) requiere una acción compleja y coordinada tanto a nivel estatal -como parte de las políticas internas- así como a nivel internacional, y el desarrollo de un modelo eficaz de la gobernanza de la seguridad alimentaria para el futuro.

Los esfuerzos por desarrollar soluciones regionales al problema del hambre y la desnutrición relacionados con la implementación de los ODM y ODS son particularmente notables en América Latina. A partir de la crisis alimentaria de los años 2007/2008, estos esfuerzos se han intensificado, convirtiéndose en un elemento integral de los debates políticos, así como en nuevas iniciativas y regulaciones legales (Piñeiro et al. 2010). Las organizaciones regionales tienen un papel especial a este respecto, ya que a través de la promoción de la integración regional contribuyen a la intensificación de la cooperación en esta área.

El objetivo de este artículo es analizar la gobernanza de la seguridad alimentaria en América Latina con especial énfasis en el papel de las organizaciones regionales y mostrar que a lo largo de los años ha sido posible desarrollar un modelo de gobernanza en la región basado en el mecanismo de cooperación Sur-Sur, promoviendo acciones conjuntas de los países en desarrollo para resolver el problema del hambre y desnutrición. La investigación abarca el periodo relacionado con la implementación de la Agenda 2030, en la cual la cooperación Sur-Sur ha sido reconocida como un instrumento importante para alcanzar los Objetivos de Desarrollo Sostenible. El análisis se basa en dos supuestos: en primer lugar, debido a la multidimensionalidad de la seguridad alimentaria, la importancia de la cooperación y las soluciones multilaterales está creciendo, y en segundo lugar, el modelo de cooperación Norte-Sur que domina la política de desarrollo internacional, conectando países altamente desarrollados y en desarrollo, se está volviendo insuficiente frente a nuevos desafíos y es necesario complementarlo con otras configuraciones, lo que, a su vez, conduce a un aumento de la importancia de la cooperación Sur-Sur.
La gobernanza de la seguridad alimentaria en América Latina: desde la cooperación Norte-Sur hacia la cooperación Sur-Sur

Katarzyna Marzęda-Młynarska

\footnotetext{
1 En inglés "stability", en el sentido del acceso continuo.
} 


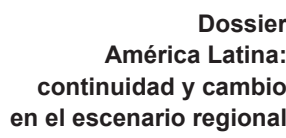

Dossier en el escenario regional
El artículo consta de tres partes. En la primera, se explican los conceptos de la gobernanza de la seguridad alimentaria y la cooperación Sur-Sur, ya que son esenciales para efectuar nuestro análisis. En la segunda parte, se presentan datos sobre la seguridad alimentaria en América Latina, con especial énfasis en amenazas y desafíos. La tercera parte está dedicada a las acciones emprendidas dentro del marco de la cooperación Sur-Sur en América Latina para proporcionar la seguridad alimentaria (objetivo 2 - ODS).

La investigación se basó en datos estadísticos e informes publicados por varias organizaciones internacionales, que incluyen: FAO, Comisión Económica para América Latina y el Caribe (CEPAL), Asociación Latinoamericana de Integración (ALADI), la Secretaría General Iberoamericana (SEGIB) y la Oficina de las Naciones Unidas para la Cooperación Sur-Sur (UNOSSC). El material recogido se sometió al análisis cuantitativo y cualitativo. El análisis cuantitativo utilizó datos sobre el número y el valor de las iniciativas para la implementación de ODS - 2 emprendidas dentro del marco de la cooperación Norte-Sur y Sur-Sur, para comparar y determinar su dinámica (tendencias al alza y a la baja). El análisis cualitativo examinó la estructura de las entidades involucradas en la cooperación y sus relaciones mutuas para capturar las regularidades existentes, lo cual permitió formular una conclusión sobre el desarrollo de un modelo regional de gobernanza de la seguridad alimentaria en América Latina.

\section{La naturaleza de la gobernanza de la seguridad alimentaria y la cooperación Sur-Sur}

Según la definición de la FAO, la gobernanza de la seguridad alimentaria significa un proceso de toma de decisiones (Marzęda-Młynarska 2014) que incluye principios y procesos formales e informales a través de los cuales se articulan los intereses, así como se toman, implementan y hacen cumplir las decisiones sobre la seguridad alimentaria en los países en nombre de los miembros de la sociedad (FAO 2011). La gobernanza entendida de esta manera incluye: en primer lugar, planificación, toma de decisiones e implementación transparentes, inclusivas y receptivas dentro de las cuatro dimensiones de la seguridad alimentaria; en segundo lugar, instituciones eficientes, efectivas, transparentes y responsables; en tercer lugar, respeto a la ley, igualdad y justicia en la asignación de recursos y prestación de servicios; y en cuarto lugar, políticas, instituciones y actividades coherentes y coordinadas (FAO 2011).

El concepto de la gobernanza de la seguridad alimentaria se desarrolló desde el comienzo de la existencia de la FAO (Shaw 2007), pero fue solo en la década de 1990, junto con los cambios que tuvieron lugar en el entorno internacional y en el ámbito de la seguridad alimentaria, que se convirtió en objeto de un interés más amplio de los investigadores. Este concepto se define de varias maneras (Candel 2014), pero el denominador común de la 
mayoría de las definiciones es que se reconoce como un mecanismo para resolver el problema de la falta de la seguridad alimentaria. Por lo tanto, no es un procedimiento bien establecido y "decretado”, sino más bien un conjunto de acciones prácticas para garantizarla en muchos niveles. Es importante destacar que la naturaleza compleja de la seguridad alimentaria significa que este problema es en gran medida incontrolable para actores individuales o mecanismos tradicionales. No puede ser tratada aisladamente de otros problemas (demográficos, ecológicos, materias primas), porque requiere soluciones integrales, la participación de muchas entidades que operan en varios niveles y dentro de diversas estructuras, y la coordinación de actividades en diversos sectores (Marzęda-Młynarska 2014: 120). Esto significa que la gobernanza de la seguridad alimentaria requiere no solo la consideración de muchos factores diferentes presentes en varios niveles y dentro de las diferentes dimensiones de la seguridad alimentaria (Pèrez-Escamilla, Shamah-Levy, Candel 2017), sino también cambios en la lógica de la operación. Si bien el Estado sigue siendo el actor básico responsable de garantizar la seguridad alimentaria a nivel nacional, una serie de restricciones y barreras resultantes de la naturaleza integral de la seguridad alimentaria hacen que la cooperación multilateral sea una necesidad.

La cooperación internacional, como mecanismo de gobernanza de la seguridad alimentaria, se ha convertido en un determinante importante de las acciones para el logro de los Objetivos de Desarrollo del Milenio y, actualmente, los Objetivos de Desarrollo Sostenible, especialmente en el contexto de la implementación del objetivo 2, relativo a la eliminación del hambre en el mundo. En la Agenda de Desarrollo de las Naciones Unidas 2030, la cooperación de los socios fue reconocida como un elemento básico de su implementación (UN 2015a), y la gobernanza eficiente de la seguridad alimentaria fue una condición clave para lograr los otros objetivos (Pèrez-Escamilla, Shamah-Levy, Candel 2017: 69). A este respecto, se prestó atención no solo al aumento de la eficacia de la cooperación en el sistema Norte-Sur, sino también a la promoción y el desarrollo de la cooperación en el sistema Sur-Sur, en función de los objetivos y experiencias comunes de los países en desarrollo. Esto se expresó en la Agenda de Acción de Addis Abeba, adoptada en la Conferencia de las Naciones Unidas sobre la Financiación para el Desarrollo en junio de 2015 (UN 2015b). Así pues, la gobernanza de la seguridad alimentaria como mecanismo para resolver el problema de la falta de la seguridad alimentaria se ha relacionado con la promoción de la cooperación Sur-Sur.

El concepto mismo de la cooperación Sur-Sur está asociado con el movimiento de países no alineados y el establecimiento del grupo G77 (Gómez Ramírez 2019). Sin embargo, se considera que el momento real de su creación es el Plan de Acción de Buenos Aires (PABA) adoptado en 1978, durante la primera Conferencia de las Naciones Unidas sobre la Cooperación Técnica de los Países en Desarrollo. Se reconoció entonces que, ante la profundización de las divisiones mundiales y los crecientes problemas económicos, el des-
La gobernanza de la seguridad alimentaria en América Latina: desde la cooperación Norte-Sur hacia la cooperación Sur-Sur

Katarzyna Marzęda-Młynarska 
Dossier América Latina: continuidad y cambio en el escenario regional envolvimiento de la cooperación entre los países en desarrollo podría tener efectos positivos, tanto al aumentar su participación en la economía global, como al apoyar la independencia para encontrar soluciones a los problemas de desarrollo de acuerdo con sus propias aspiraciones, valores y necesidades (PABA 1978). El PABA propuso un cambio en la lógica de la cooperación internacional para el desarrollo y una transición de un modelo vertical, basado en las relaciones entre los países altamente desarrollados (donantes) y los países en desarrollo (receptores), al modelo horizontal: países en desarrollo (donantes, receptores) -países en desarrollo (donantes, receptores). Además, “allanó el camino para cambios importantes en el enfoque de la asistencia para el desarrollo, considerándola un proceso multidimensional, y también mostró a los países en desarrollo un modelo de cooperación compatible con sus capacidades, el respeto a la soberanía nacional, la independencia económica y no injerencia en los asuntos internos" (Gómez Ramírez 2019: 3).

La adopción de la Agenda 2030, en 2015, marcó una nueva etapa en el desarrollo de la cooperación Sur-Sur, que se manifestó en el reconocimiento de su importante papel en la implementación de los ODS (UN 2015a), confirmado durante la Segunda Conferencia de las Naciones Unidas sobre Cooperación Sur-Sur en Buenos Aires en marzo de 2019. El documento final de esta conferencia enfatizó que la cooperación Sur-Sur conduce a oportunidades de desarrollo más diversas y no debe verse como asistencia oficial para el desarrollo. Su programa debe ser determinado por los países del Sur, guiados por los principios de respeto a la soberanía nacional, igualdad y falta de condicionalidad o interferencia en asuntos internos, beneficio mutuo y carácter voluntario. Además, debe basarse en objetivos compartidos y solidaridad (UN 2019).

Según la Oficina de las Naciones Unidas para la Cooperación Sur-Sur, la cooperación Sur-Sur es un marco amplio para la cooperación entre los países del Sur en el área de política, economía, sociedad, cultura, protección ambiental y tecnología, y puede tener el carácter bilateral, regional, intrarregional o interregional, confiar en el intercambio de conocimientos, habilidades y recursos para la implementación conjunta de los objetivos de desarrollo y adoptar la forma de un mayor intercambio comercial, flujos de inversión extranjera directa, integración regional, transferencia de tecnología, intercambio de soluciones y expertos, así como otras formas de cooperación (UNOSSC 2019). A este respecto, se asigna un papel especial a la cooperación regional Sur-Sur, cuyo objetivo es fortalecer el desarrollo y/o la integración de la región. Según la definición, es una forma de cooperación entre países en desarrollo en la que las relaciones entre sus participantes están reguladas por un mecanismo institucional formalmente reconocido por todas las partes y llevado a cabo a través de programas y proyectos (SEGIB 2018). Se reconoce que, debido a la proximidad geográfica de los miembros, un pasado similar y problemas comunes, las organizaciones regionales están mejor preparadas para enfrentar nuevos desafíos. Por esta razón, su actividad a menudo se ve como un complemento de las acciones tomadas a nivel global. 


\section{Amenazas y desafíos para la seguridad alimentaria en América Latina}

La seguridad alimentaria es un fenómeno amplio y complejo, afectado por una variedad de factores. Primero, las fuerzas de la naturaleza, es decir, el clima, la disponibilidad de agua y tierra. En segundo lugar, el poder económico que depende de los niveles de ingresos, los precios de los alimentos y la situación en el mercado. Tercero, las fuerzas sociales, incluidas las características demográficas y las preferencias alimentarias. En cuarto lugar, un factor tecnológico, es decir, nuevas tecnologías y conocimientos que pueden contribuir significativamente a aumentar el suministro de alimentos, y, quinto, un factor político, incluida la política agrícola y alimentaria, que se traduce directamente en el nivel de seguridad alimentaria de los Estados (MarzędaMłynarska 2014).

América Latina tiene todos los recursos y atributos ambientales para garantizar su seguridad alimentaria y convertirse en un líder mundial en producción de alimentos. Es una región rica en tierra cultivable, agua, biodiversidad, condiciones climáticas diversas y potencial comercial. Sin embargo, la multitud de factores que condicionan la seguridad alimentaria significa que este potencial "de base" es insuficiente para garantizarla y el nivel de seguridad alimentaria se caracteriza por una alta variabilidad. Esto significa que los cambios positivos que ocurren en un periodo dado no pueden ser tratados como tendencias permanentes para el futuro. Tales conclusiones, entre otras, se pueden sacar de informes que resumen la implementación de los Objetivos de Desarrollo del Milenio y los Objetivos de Desarrollo Sostenible. Sus datos indican que, después de un período de disminución prolongada, el número de personas en riesgo de inseguridad alimentaria ha aumentado nuevamente desde el año 2016. Mientras que en 2015 el número de personas hambrientas y desnutridas en el mundo se estimó en 777 millones (UN 2015c), en 2017 aumentó a 821 millones (UN 2018). También en América Latina, el número de hambrientos y desnutridos aumentó de 38,9 millones en 2016 a 39,3 millones en 2017 (FAO 2018).

Sin embargo, al evaluar la situación a largo plazo, debe tenerse en cuenta que se han logrado avances significativos en el área de la seguridad alimentaria en América Latina a lo largo de los años. Se ha conseguido reducir a la mitad el porcentaje de personas hambrientas y desnutridas, del 14,7\% en 1990-92 al 5,5\% en 2014-16, de acuerdo con el objetivo 1c establecido en el marco de los Objetivos de Desarrollo del Milenio (UN 2015c: 21). El mayor progreso en esta área se registró en la subregión de América del Sur, donde el porcentaje cayó por debajo del 5\%. En América Central, el progreso fue mucho más lento y, finalmente, la tasa disminuyó hasta el 6,6\%. Por otro lado, la situación en la subregión del Caribe fue la menos satisfactoria a este respecto, ya que allí el porcentaje de personas hambrientas y desnutridas disminuyó del $27 \%$ en 1990-92 al 19,7\% en 2014-16 (UN 2015c: 21). Generalmente, en este con-
La gobernanza de la seguridad alimentaria en América Latina: desde la cooperación Norte-Sur hacia la cooperación Sur-Sur

Katarzyna Marzęda-Młynarska 
Dossier América Latina: continuidad y cambio en el escenario regional texto, los factores económicos, incluido el crecimiento económico dinámico y la disminución de la pobreza, fueron decisivos (UN 2015c: 21). Al analizar la situación en América Latina en términos de las dimensiones de la seguridad alimentaria, debe tenerse en cuenta que en la mayoría de ellos se ha logrado un progreso significativo. En el caso de la disponibilidad física de alimentos, el factor responsable de su suministro fue principalmente la producción. Como región, América Latina es un exportador neto y un importante proveedor de alimentos para el mercado mundial. Con los años, la producción agrícola en la región creció más rápidamente que la población, lo que contribuyó no solo al aumento de la disponibilidad de alimentos, sino también a la cantidad de calorías producidas por persona. (CEPAL 2016: 16). La mejora de la situación en esta dimensión tuvo un impacto positivo en la diversidad de la dieta. Aunque todavía se basa en cereales, la proporción de carne, productos lácteos, así como verduras y frutas está creciendo (CEPAL 2016: 15).

También se han observado cambios positivos en términos de disponibilidad económica de alimentos, aunque la pobreza sigue siendo un problema grave y una barrera importante para garantizar la seguridad alimentaria en la región. América Latina tiene las mayores disparidades de ingresos medidas por el índice de Gini, lo que tiene un impacto negativo en la situación de las personas más pobres. Aunque en los años 1990-2012 la pobreza se redujo significativamente, lo que fue acompañado por una lenta disminución de la desigualdad de ingresos, todavía afecta a aproximadamente el $30 \%$ de la población. Por otro lado, los precios de los alimentos tuvieron un impacto significativo en la mejora de la disponibilidad económica de los alimentos, ya que -después de un período de crecimiento en 2007/8 y 2010/11-comenzaron a disminuir. Sin embargo, debe enfatizarse que, si bien la disminución de los precios de los alimentos a nivel mundial tuvo un impacto positivo en su disponibilidad económica en la región, este efecto no se distribuyó de manera uniforme en todos los países. En algunos, fue neutralizado por factores relacionados con la situación económica interna (CEPAL 2016: 19).

En cuanto a la salud, se han logrado avances indudables en la lucha contra la desnutrición resultante de la falta de alimentos, así llamado "subconsumo". El porcentaje de niños menores de 5 años que tuvieron retraso en el crecimiento y bajo peso asociado con deficiencias de nutrientes en los alimentos - considerado como un indicador de desnutrición- en América Latina cayó del $24,5 \%$ en 1990 al $11,3 \%$ en 2015. Sin embargo, al mismo tiempo hubo un aumento significativo en la obesidad, tanto en niños como en adultos (CEPAL 2016: 19). Esto significa que el "subconsumo" característico para las regiones en desarrollo, asociado con una dieta muy baja en calorías y oligoelementos, está actualmente acompañado por el fenómeno del "consumo excesivo", característico de los países altamente desarrollados, y que es el resultado de una dieta demasiado alta en calorías.

Con respecto a la cuarta dimensión, es decir, la estabilidad del acceso, debe tenerse en cuenta que América Latina no está incluida en las regiones en ries- 
go de inseguridad alimentaria permanente. Los problemas con su provisión que aparecieron a lo largo de los años fueron de naturaleza temporal y se asociaron con fenómenos periódicos como crisis económicas, desastres naturales, fenómenos climáticos extremos, así como conflictos e inestabilidad política. Sin embargo, hay que tener en cuenta que esta situación puede cambiar, ya que esta región está muy expuesta a los efectos del cambio climático, que en el futuro puede tener un impacto negativo tanto en la producción de alimentos como en los ingresos de los residentes, así como en la disponibilidad de recursos naturales o redes de suministro (FAO 2017).

La estabilidad de la disponibilidad de alimentos está inseparablemente asociada a los desafíos y amenazas de la seguridad alimentaria. De manera similar a los factores condicionantes, los desafíos y amenazas se pueden analizar en cuatro grupos: como amenazas y desafíos ambientales (cambio climático, déficit de agua, acceso a tierras cultivables), socioeconómicos (forma de distribución de alimentos, crecimiento de la población, aumento de precios de los alimentos, cambios en las tendencias nutricionales), los relacionados con el progreso tecnológico (industrialización de la agricultura, OMG, ingeniería genética, derechos de propiedad intelectual) y de carácter político (conflictos, inestabilidad política). Algunos de ellos son periódicos y tienen un impacto temporal en el nivel de seguridad alimentaria de los países, mientras que otros, como las amenazas ambientales, condicionan permanentemente la posibilidad de garantizar la seguridad alimentaria.

Según los autores del Global Food Safety Index, publicado anualmente, el mayor impacto en el nivel de la seguridad alimentaria en 2018 tuvieron: cambios climáticos, riesgos financieros, es decir, aumento de precios de los alimentos, falta de soluciones de seguridad social relacionada con los alimentos, financiamiento limitado y falta de seguros para los agricultores, riesgos relacionados con el comercio, es decir, proteccionismo comercial, restricciones infraestructurales y riesgos políticos: conflictos, inestabilidad política, corrupción (GFSI 2018: 8).

La mayoría de los expertos están de acuerdo en que el cambio climático es la mayor amenaza para la seguridad alimentaria en América Latina (FAO 2017; GSFI 2018; IFPRI 2018; CEPAL, FAO, ALADI 2016). Esto se debe a tres razones. En primer lugar, el papel dominante del sector agrícola en la economía de los países de la región, en segundo lugar, el bajo potencial de adaptación al cambio climático y, en tercer lugar, la ubicación geográfica. Las previsiones de la FAO apuntan, sobre todo, a cambios radicales en las condiciones ambientales, incluido el acceso al agua, la calidad del suelo, la temperatura, la aparición de enfermedades y plagas y la gravedad de los fenómenos meteorológicos extremos. Todos estos cambios afectarán significativamente la disponibilidad física y económica de los alimentos, la calidad de la salud y la estabilidad del acceso (FAO 2017: 6). En el caso de la disponibilidad física de alimentos, se llama atención al impacto directo del cambio climático en la producción (agricultura), en la forma de su reducción potencial y la re-
La gobernanza de la seguridad alimentaria en América Latina: desde la cooperación Norte-Sur hacia la cooperación Sur-Sur

Katarzyna Marzęda-Młynarska 
Dossier América Latina: continuidad y cambio en el escenario regional ducción de la variedad de alimentos producidos, las condiciones de almacenamiento, incluido el aumento del riesgo de pérdidas, y el desplazamiento de las zonas de producción de materias primas agrícolas básicas en la región, como el café, patatas, caña de azúcar o maíz. En el caso de la disponibilidad económica de alimentos, el principal desafío es la reducción de los ingresos de las personas que viven de la agricultura y el posible aumento de precios de los alimentos. Los expertos también señalan que, si las condiciones climáticas extremas se intensifican, la infraestructura local puede dañarse y, por lo tanto, el suministro de alimentos puede deteriorarse en el mercado interno. Los cambios climáticos y los fenómenos relacionados a ellos también tendrán un impacto en la calidad sanitaria de los alimentos, incluida una reducción en la inocuidad alimentaria debido al deterioro del saneamiento y la disponibilidad limitada de agua (FAO 2016: 6-7).

La investigación realizada en 2008-2015 por la FAO sobre la dimensión económica de los cambios climáticos en los países de América Latina mostró su impacto potencialmente negativo principalmente en la agricultura. Se ha reconocido que serán más notorios en la subregión centroamericana que reúne al 60\% de los agricultores de la región, donde un aumento en la temperatura promedio puede significar una disminución en la producción de dos productos alimenticios básicos: maíz y frijoles (CEPAL, FAO, ALADI 2016: 76). Además de la agricultura, un sector importante desde el punto de vista de la seguridad alimentaria amenazado por los cambios climáticos es la acuicultura. Se estima que, como resultado del calentamiento global y la acidificación de aguas de los océanos, así como los fenómenos climáticos extremos, las áreas costeras utilizadas en la acuicultura disminuirán, el alcance de pesquerías cambiará a favor de latitudes más altas, igual que la ubicación de los puertos (CEPAL, FAO, ALADI 2016: 81).

Los fenómenos meteorológicos extremos son una fuente importante de amenazas a la seguridad alimentaria en América Latina relacionadas con el cambio climático. Los datos indican que fenómenos de este tipo se han intensificado con los años, al igual que el número de población afectada por ellos (CEPAL, FAO ALADI 2016: 82). La agricultura es un sector particularmente vulnerable a sus efectos negativos. Los análisis de la Comisión Económica de las Naciones Unidas para América Latina y el Caribe muestran que solo como resultado del fenómeno del Niño, que afectó a muchos países de la región, las pérdidas agrícolas representaron casi el 31\% de todas las pérdidas asociadas con este fenómeno en los años 1997-98 (CEPAL, FAO, ALADI 2016: 85)

Los otros tres grupos de riesgo identificados por GFSI 2018 no tienen un impacto tan complejo y a largo plazo en la seguridad alimentaria en América Latina, pero pueden determinar periódicamente su nivel. La sensibilidad de la región al aumento de precios mundiales de los alimentos depende en gran medida del contexto individual, incluida la posición de cada país en el comercio internacional. Sin lugar a duda, un factor que aumenta el nivel 
de sensibilidad de los países a la volatilidad de los precios es la especialización en la producción y exportación de materias primas agrícolas seleccionadas. Según los expertos de la CEPAL, para limitarlo es necesario desarrollar la integración económica y el comercio en la región, no solo contribuyendo a la reducción de los costos de transacción, sino también a la creación de canales locales de distribución de alimentos que conecten a los productores y consumidores locales (CEPAL 2016: 40). Sin embargo, un nivel relativamente alto de proteccionismo comercial puede ser un desafío importante a este respecto. Si bien, en general, América Latina en su conjunto ha logrado liberalizar significativamente el comercio intrarregional de productos alimenticios, existen diferencias significativas a este respecto en el comercio entre subregiones individuales. Un ejemplo de esto es la falta de acuerdo sobre la liberalización del comercio entre los países asociados en la Comunidad del Caribe (CARICOM) y los países de la subregión sudamericana, o MERCOSUR y los países de América Central y México (CEPAL 2016: 60-62).

Otra forma de reducir el impacto negativo de los precios mundiales en la seguridad alimentaria local puede ser cambiar la estructura de los alimentos producidos y pasar de la exportación de materias primas agrícolas y pesqueras a la exportación de alimentos sofisticados y de alta calidad. Cabe destacar que la región de América Latina, cuya participación en las exportaciones mundiales de alimentos es del 15\%, tiene un potencial significativo a este respecto y en el futuro puede aumentar su papel como proveedor de alimentos en el mercado global (CEPAL 2016: 51).

Sin lugar a duda, la pobreza y las persistentes desigualdades de ingresos son un desafío importante para garantizar la seguridad alimentaria en América Latina. Si bien, desde principios de 2000, se han logrado avances significativos en la reducción de las disparidades de ingresos de hogares e individuos, así como en la distribución de ingresos, ambos procesos se desaceleraron en 2014 (CEPAL 2018: 19). Casi el 30\% de la población de la región vive en la pobreza, de los cuales casi el $10 \%$ en la pobreza extrema. Este problema afecta principalmente a los habitantes de las zonas rurales, donde la escala de pobreza es un 20\% más alta que en las ciudades (CEPAL 2018: 20), así como a los pueblos indígenas y descendientes de esclavos africanos.

Además de los ingresos provenientes de empleo y jubilación, las transferencias a los hogares más pobres juegan un papel importante en la lucha contra la pobreza, incluidas las prestaciones sociales y otras formas de aumentar la disponibilidad de alimentos, como los Programas de Transferencias Monetarias Condicionadas (TMC), las pensiones sociales y la financiación de comidas escolares. Sin embargo, el potencial de este tipo de programas, especialmente las TMC, no se está utilizando en su plena capacidad debido a problemas relacionados con la identificación de los receptores de ayuda, lo que resulta en la exclusión de una parte importante de la población que experimenta escasez. Los programas de este tipo se implementan solo en 20 de los 33 países de la región y cubren solo el 20\% de la población de la región (FAO,
La gobernanza de la seguridad alimentaria en América Latina: desde la cooperación Norte-Sur hacia la cooperación Sur-Sur

Katarzyna Marzęda-Młynarska 
Dossier América Latina: continuidad y cambio en el escenario regional
PAH, WHO 2017: 66). A pesar de que, en toda América Latina, el gasto social se mantuvo estable en relación con el gasto público total y creció más rápido que la producción en 2015-2016, que fue un elemento importante en la lucha contra la pobreza, sin embargo, la financiación de la política social sigue siendo un desafío importante en América Latina, también en el contexto de la seguridad alimentaria (FAO, PAH, WHO 2017: 66-67).

\section{Actividades realizadas en el marco de la cooperación Sur-Sur como parte de la gobernanza regional de la seguridad alimentaria en América Latina}

La conciencia de la importancia de la seguridad alimentaria y la necesidad de alcanzar los Objetivos de Desarrollo del Milenio (objetivo 1c) y también los Objetivos de Desarrollo Sostenible (objetivo 2) han contribuido no solo a la dinamización de la cooperación internacional y la introducción de problemas de hambre y desnutrición en la agenda regional, sino también a la construcción de un enfoque regional para gestionar este problema en América Latina. El marco para su elaboración fue creado por dos iniciativas regionales. La primera, del año 2005, se propuso la eliminación total del hambre en la región para 2025 y fue el primer acuerdo regional de este tipo, así como constituyó la base de la Iniciativa América Latina y Caribe sin Hambre (IALCSH) adoptada por todos los países de la región. La segunda, del año 2015, adoptada en el marco de la Comunidad de Estados Latinoamericanos y Caribeños (CELAC), como el Plan de Seguridad Alimentaria, Nutrición y Erradicación del Hambre de la CELAC 2025, contribuyó integrar el proceso político e insertar la seguridad alimentaria en la agenda regional de los países de América Latina y el Caribe (CEPAL 2016: 89).

Ambas iniciativas propusieron una nueva perspectiva sobre el problema de la seguridad alimentaria y soluciones para garantizarla. La iniciativa del 2005 brindó la oportunidad de debatir sobre este tema en dos niveles simultáneamente: regional -dentro de las organizaciones subregionales, es decir, la Unión de Naciones Suramericanas (UNASUR), el Mercado Común del Sur (MERCOSUR), y el Sistema de Integración de Centroamérica (SICA); y nacional -dentro del marco de las instituciones y mecanismos establecidos por los Estados. A su vez, la iniciativa del 2015 confirmó el apoyo de los países de la región para incluir el hambre y la desnutrición en la cooperación regional, lo que, a su vez, les permitió abordar esta cuestión de una manera más integral y compleja (CEPAL 2016: 89).

Según los expertos, el elemento clave del nuevo enfoque para la gobernanza de la seguridad alimentaria en América Latina fue la transferencia de la cuestión del hambre y la desnutrición de una esfera puramente técnica a la esfera política y el ajuste estratégico de los criterios técnicos a los objetivos polí- 
ticos, como parte de un enfoque integrado (CEPAL 2016: 89). Como resultado de un largo proceso político, se desarrolló un enfoque regional basado en tres elementos: 1) la inclusión de la seguridad alimentaria en la agenda regional oficial; 2) el modelo regional de gobernanza de la seguridad alimentaria; y 3 ) la institucionalización de la seguridad alimentaria a nivel nacional en forma de instrumentos jurídicos y políticos (CEPAL 2016: 89-90).

El modelo latinoamericano de gobernanza de la seguridad alimentaria significó, en primer lugar, un cambio en la forma de decidir sobre asuntos relacionados con la seguridad alimentaria y la implementación de los ODS, al expandir y complementar los mecanismos tradicionales de toma de decisiones con nuevas formas institucionalizadas de toma de decisiones y mecanismos de participación política, teniendo en cuenta un número creciente de partes interesadas. En segundo lugar, conllevó la elaboración y la implementación de mecanismos de coordinación intersectorial y la creación de políticas integradas, incluidas medidas interdisciplinarias para la implementación y evaluación de la seguridad alimentaria, al reconocer que el hambre y la desnutrición constituyen un problema multisectorial. En tercer lugar, adoptó la lógica de "doble vía" en la implementación de políticas de seguridad alimentaria, incluidas medidas a corto plazo para garantizar una respuesta inmediata al hambre y la desnutrición, y medidas de desarrollo sostenible a mediano y largo plazo para garantizar la estabilidad de la seguridad alimentaria (CEPAL 2016: 89).

Un elemento clave de la gobernanza de la seguridad alimentaria en América Latina es la cooperación intrarregional en el marco de la fórmula Sur-Sur. Se observaron efectos positivos de este tipo de cooperación, especialmente en el contexto de la implementación de los Objetivos de Desarrollo del Milenio, enfatizando los beneficios del intercambio de experiencias y la cooperación de los Estados y las organizaciones subregionales. Actualmente, la cooperación Sur-Sur es la base para la implementación de la Agenda 2030 y los Objetivos de Desarrollo Sostenible.

Como región, América Latina se convirtió en el líder de la cooperación SurSur. Esto se evidencia no solo por la cantidad de proyectos, que crecen de año en año, sino también por el número de instituciones y organismos regionales involucrados en la promoción de este tipo de cooperación (Grupo de Trabajo de Cooperación Internacional de CELAC, Grupo de Trabajo de Alto Nivel de UNASUR sobre Cooperación Sur-Sur; Grupo sobre Cooperación Internacional MERCOSUR, Comité de Cooperación Sur-Sur de la CEPAL). Además, en la Cumbre Iberoamericana de 2008, se adoptó el Programa Iberoamericano para el Fortalecimiento de la Cooperación Sur-Sur, cuyo objetivo era aumentar la capacidad institucional y promover esta forma de cooperación. Los datos sobre este tema se han recopilado y publicado desde 2007 en los informes de la Secretaría General Iberoamericana (SEGIB 2018a: 20).

Cabe destacar que la cooperación Sur-Sur ha sido reconocida oficialmente como un instrumento para implementar la Agenda 2030. Su objetivo principal
La gobernanza de la seguridad alimentaria en América Latina: desde la cooperación Norte-Sur hacia la cooperación Sur-Sur

Katarzyna Marzęda-Młynarska 


\begin{abstract}
Dossier
América Latina:

continuidad y cambio en el escenario regional
\end{abstract}

Gráfico 1.

Los flujos de la AOD globales y hacia la región

de América Latina (en millones de dólares americanos)
Flujos de Ayuda Oflclal al Desarrollo (AOD) globales y hacla los paises Iberoamerlcanos

Expresados en millones de dólares; precios constantes, 2015) Fuente: OECD (stats.oeod.org/). Datos consultados en febrer de ao18.
The flow of Offlclat

Development AssIstance (ODA), globally and to Ibero-Amerlcan natlons

(Millions of dollars, at 2015 constant prices) (Mundial - Worldwide, América Latina - Latin America) Source: OECD (stats.oecd.org/). Data consulted in February, ao18.
AOD Paises en desarrollo ODA Developing Countries ODA Latin America

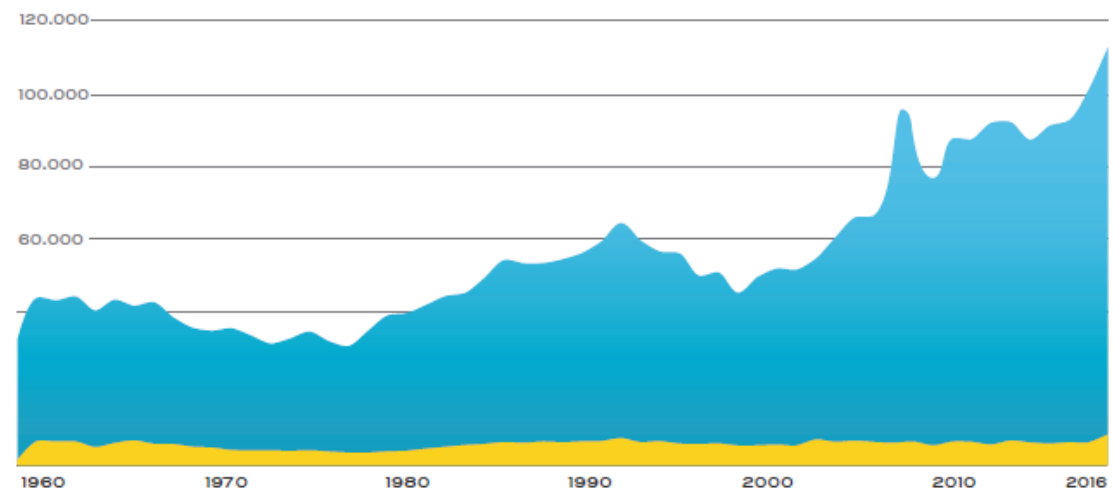

Fuente: SEGIB 2018a, p. 59.

es aumentar las capacidades (organizativas, administrativas, institucionales, financieras) de los Estados para implementar los ODS. Aunque la cooperación bilateral sigue siendo la forma dominante, la importancia de la cooperación regional está creciendo con la reducción de las iniciativas de cooperación con la participación de donantes de países desarrollados. Mientras que, en 2001, el 10,1\% de los fondos de la Asistencia Oficial para el Desarrollo (AOD) se destinó a países latinoamericanos, en 2010 este índice disminuyó al 4,1\% (SEGIB 2018a: 58). La disminución de la financiación para la cooperación al desarrollo en el marco de la AOD (Gráfico 1) dio como resultado el retorno a la idea de la cooperación Sur-Sur y la búsqueda de formas alternativas de apoyo al desarrollo.

La disminución del número de iniciativas en la fórmula Norte-Sur también es una manifestación de un cambio en el enfoque relacionado con a la asistencia para el desarrollo en la política internacional dominada por donantes de países altamente desarrollados. Los expertos latinoamericanos señalan que el sistema actual de asistencia oficial para el desarrollo, basado en el PIB per cápita, no satisface las necesidades de los países de la región, ya que la mayoría de ellos están en el grupo de países con un PIB medio bajo o medio alto. Además, el sistema de la AOD no tiene en cuenta criterios distintos del PIB que reflejan la complejidad y la multidimensionalidad de los problemas de desarrollo de los países de la región (SEGIB 2018b: 29). Tam- 
bién se reconoce que la disminución de la importancia de la cooperación Norte-Sur está asociada con su condicionamiento, es decir, con el requisito de que los países receptores adopten principios y valores específicos. Ante la disminución del interés de los países en desarrollo en la cooperación clásica Norte-Sur, los países altamente desarrollados están tratando de mantener su posición dominante en la política internacional de desarrollo de otra manera. La cooperación triangular se está convirtiendo en un nuevo canal de cooperación Norte-Sur, permitiendo a los países altamente desarrollados influir en la cooperación Sur-Sur y configurarla de acuerdo con sus propios intereses (Abdenur, Da Fonseca 2013). El análisis de los datos estadísticos sobre la cooperación trilateral y bilateral en la fórmula Sur-Sur indica su alta dinámica medida por el índice de variabilidad en el número de iniciativas anuales (Tabla 1). En el caso de la cooperación trilateral, después de aumentos iniciales del 75\% en promedio en 2010 y 2011, se registró una disminución significativa en 2012. Aunque desde 2013 el número de iniciativas se ha estabilizado en 160 por año (en promedio), ha seguido una tendencia a la baja en 2015 y 2016. En el caso de la cooperación bilateral Sur-Sur, excluyendo los años 2009-2011, cuando se registraron cambios significativos en el número de iniciativas, su dinámica permanece en un nivel estable.

\begin{tabular}{lcccccccc}
\hline & 2009 & 2010 & 2011 & 2012 & 2013 & 2014 & 2015 & 2016 \\
\hline $\begin{array}{l}\text { Cooperación Sur-Sur: } \\
\text { Número }\end{array}$ & 881 & 529 & 815 & 709 & 978 & 885 & 1206 & 1105 \\
\cline { 2 - 8 } \begin{tabular}{l} 
Dinámica (año a año) \\
\cline { 2 - 7 }
\end{tabular} & $-39 \%$ & $+54 \%$ & $-13 \%$ & $+37 \%$ & $-9 \%$ & $+36 \%$ & $-8 \%$ \\
\hline $\begin{array}{l}\text { Cooperación Sur-Sur: } \\
\begin{array}{l}\text { Número } \\
\text { Dinámica (año a año) }\end{array}\end{array}$ & 46 & 83 & 144 & 77 & 166 & 183 & 168 & 149 \\
\cline { 2 - 8 } & & $+80 \%$ & $+73 \%$ & $-46 \%$ & $+115 \%$ & $+10 \%$ & $-8 \%$ & $-11 \%$ \\
\hline
\end{tabular}

Fuente: Elaboración propia sobre la base de los informes SEGIB de los años 2009-2018.

Los datos acumulados de la década 2006-2015 indican que en este periodo los países latinoamericanos participaron en 7.370 programas, proyectos y actividades, la gran mayoría de los cuales, hasta el $82 \%$, se implementaron en la fórmula Sur-Sur (cooperación bilateral), el 13\% en la fórmula Norte-Sur (cooperación tripartita), y el 5\% la cooperación regional Sur-Sur (SEGIB 2018a: 67). En cuanto al contenido, la mayoría de las iniciativas se implementaron en tres sectores: salud (607), administración gubernamental (531) y agricultura (518). Durante toda la década, casi el 45\% de las iniciativas se centraron en fortalecer los sectores mencionados anteriormente, importantes para garantizar la seguridad alimentaria (SEGIB 2018a: 77). El análisis de los datos en términos de cumplimiento de los Objetivos de Desarrollo Sostenible mostró que casi 1/3 de las iniciativas implementadas en el periodo 2013-2015 se referían al objetivo 2 (hambre cero) y al objetivo 3 (protección de la salud) (SEGIB 2018a: 81).
La gobernanza de la seguridad alimentaria en América Latina: desde la cooperación Norte-Sur hacia la cooperación Sur-Sur

Katarzyna Marzęda-Młynarska

Tabla 1.

El número y la dinámica de aumento/disminución de iniciativas en el marco de la cooperación triangular y Sur-Sur en América Latina en el periodo 2009-2016 
Dossier

América Latina:

continuidad y cambio en el escenario regional
Además del área de la agricultura, de la cual la mayoría de las iniciativas formaron parte de las actividades ODS-2, también los programas y proyectos implementados en otros sectores incluyeron actividades para mejorar la seguridad alimentaria. En el caso de la protección de la salud, se referían, entre otros, a la lucha contra la desnutrición y enfermedades relacionadas, en el caso de las instituciones públicas se trataba del aumento de la eficiencia de las políticas públicas y, en el caso del acceso al agua, se referían al saneamiento y aumento de acceso al agua potable. Debido a la complejidad de la seguridad alimentaria, muchos programas y proyectos implementados en sectores individuales se asociaron directa o indirectamente con la provisión de la seguridad alimentaria.

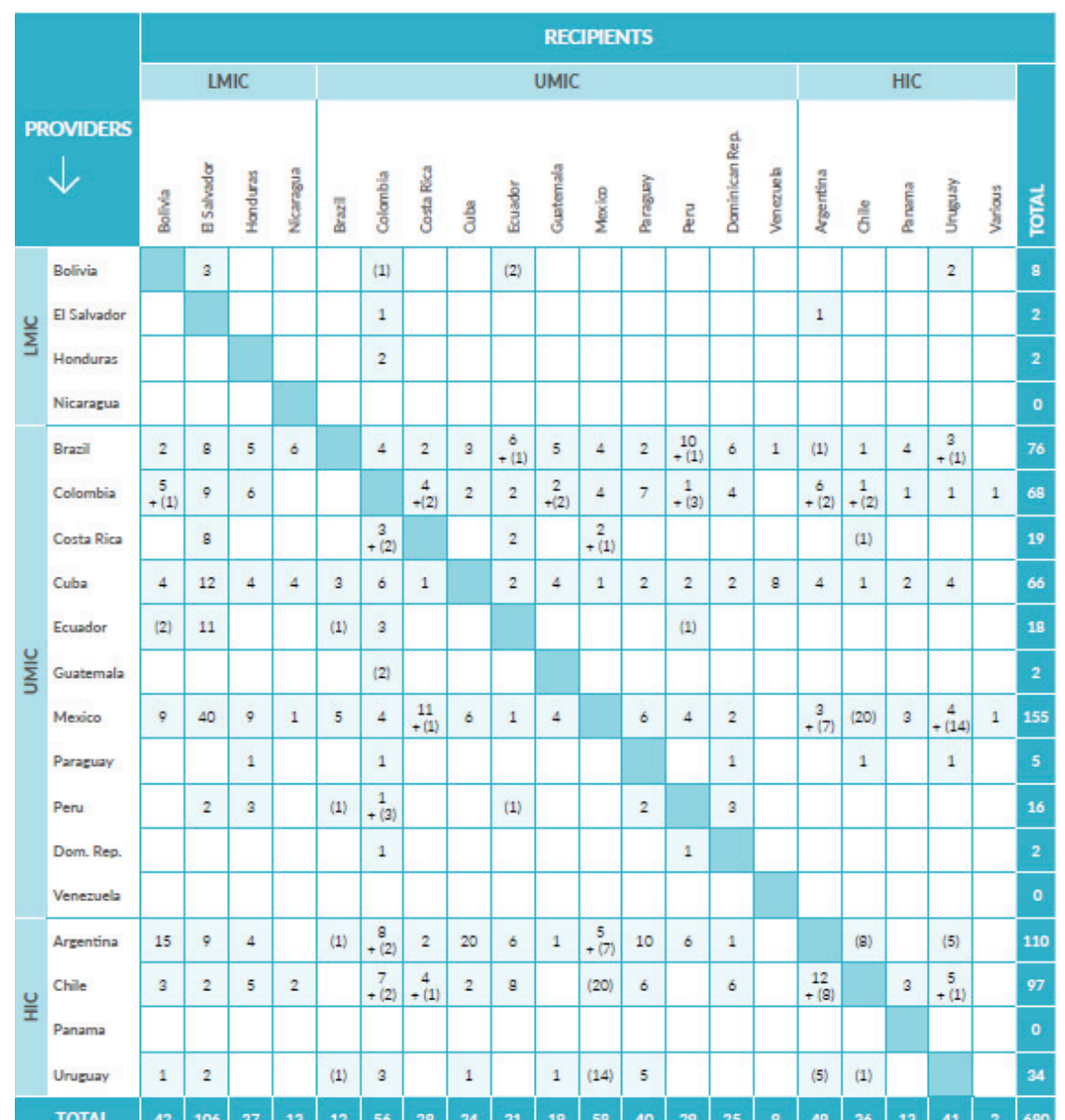

Fuente: SEGIB 2018b: 52. 
La mayoría de las iniciativas de la cooperación Sur-Sur implementadas en América Latina se refieren a la cooperación bilateral y regional. Un rasgo característico de la cooperación bilateral es la naturaleza mixta de los papeles desempeñados por los países individuales. Como indican los datos (Gráfico 2), son donantes y beneficiarios, lo cual no ocurre en el caso de la cooperación tradicional Norte-Sur.

En los años 2006-2015, entre los donantes más importantes se encontraban cuatro países: Brasil, México, Argentina y Cuba, mientras que los países receptores incluían: El Salvador, Bolivia, Costa Rica, Ecuador, Guatemala y Paraguay. Las iniciativas implementadas se centraron en cuatro sectores: cooperación en el ámbito económico y social, protección del medio ambiente y administración pública. El área dominante era la protección de la salud, incluidos los problemas de una nutrición adecuada (SEGIB 2018a).

En 2016, la mayoría, es decir, el 17,2\% de todos los proyectos, se refería a problemas de salud ampliamente entendidos. La agricultura era otra área importante de cooperación, que reunía el 12,5\% de todas las iniciativas. Cabe destacar que hasta 83 de los proyectos implementados en 2016 estaban directamente relacionados con la seguridad alimentaria y la lucha contra el hambre, es decir, el segundo ODS-Hambre cero (SEGIB 2018b: 64-65, 80). Se referían, entre otros, a la cooperación fitosanitaria y salud animal, a la seguridad alimentaria y al acceso garantizado a alimentos saludables de calidad adecuada, así como a la estabilidad del acceso, a la promoción de la agricultura y la pesca familiar, a la gestión de la diversidad genética y biológica y a la adaptación a los cambios climáticos (SEGIB 2018b: 81).

Una dimensión particular de la cooperación Sur-Sur en América Latina es la cooperación regional Sur-Sur, cuyo objetivo es fortalecer la integración de la región. Sus participantes son tanto países como organizaciones regionales, y su base constituyen los programas y proyectos pan- y subregionales implementados durante muchos años (en promedio de uno a 14 años). Unas de las organizaciones regionales más activas dentro del marco de la cooperación regional Sur-Sur en 2016 fueron: la Secretaría General Iberoamericana (SEGIB), el Sistema de Integración Centroamericana (SICA), MERCOSUR y el Banco Interamericano de Desarrollo. En cuanto a los sectores de esta cooperación, dominaron proyectos de carácter económico (pequeñas y medianas empresas, desarrollo de mercados laborales, nuevas tecnologías), infraestructura (desarrollo y modernización de infraestructura vial) y producción (agricultura, acuicultura, comercio, industria, energía). Es importante destacar que la gran mayoría de todos los proyectos implementados en 2016 se referían a los Objetivos de Desarrollo Sostenible, incluidos siete relacionados con el Objetivo 2-Hambre Cero (SEGIB 2018b: 139). En la Tabla 2 se presenta la lista de programas y proyectos implementados en 2016 como parte de la cooperación regional Sur-Sur relacionada con la seguridad alimentaria.

Cabe destacar que esta forma de cooperación regional es única a escala global y específica para América Latina. Como señalan los expertos, gracias a la recupe-
La gobernanza de la seguridad alimentaria en América Latina: desde la cooperación Norte-Sur hacia la cooperación Sur-Sur

Katarzyna Marzęda-Młynarska 


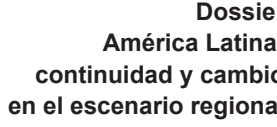

Tabla 2.

Cooperación regional Sur-

Sur - proyectos y programas relacionados con la seguridad alimentaria en 2016

\begin{tabular}{|c|c|c|}
\hline Marco institucional & Nombre & Tipo \\
\hline $\begin{array}{l}\text { Inter-American } \\
\text { Development Bank - IBD }\end{array}$ & $\begin{array}{l}\text { Improvement of Animal Production System } \\
\text { with an emphasis on dairy cattle in the Andean } \\
\text { region within the context of climate change }\end{array}$ & proyecto \\
\hline $\begin{array}{l}\text { Inter-American } \\
\text { Development Bank - IBD }\end{array}$ & $\begin{array}{l}\text { Enhancing the resilience of production system } \\
\text { to reduce vulnerability of small producers } \\
\text { through development of "forgotten" Andean } \\
\text { crops }\end{array}$ & proyecto \\
\hline Andean Community - AC & $\begin{array}{l}\text { Andean Regional Program for Strengthening } \\
\text { Meteorological, Hydrological, and } \\
\text { Climatological Services and Development } \\
\text { (PRASDES) }\end{array}$ & programa \\
\hline $\begin{array}{l}\text { Mesoamerican Program } \\
\text { (Mexico) }\end{array}$ & $\begin{array}{l}\text { Center for Climate Services in Mesoamerican } \\
\text { and the Caribbean Phase II }\end{array}$ & proyecto \\
\hline $\begin{array}{l}\text { Mesoamerican Program } \\
\text { (Mexico) }\end{array}$ & $\begin{array}{l}\text { Strengthening capacities to reduce } \\
\text { vulnerability to reduce drought in the } \\
\text { Mesoamerican region }\end{array}$ & proyecto \\
\hline $\begin{array}{l}\text { Mesoamerican Integration } \\
\text { and Development Project }\end{array}$ & $\begin{array}{l}\text { Mesoamerican Strategy for Environmental } \\
\text { Sustainability }\end{array}$ & proyecto \\
\hline $\begin{array}{l}\text { Central American } \\
\text { Economic Integration } \\
\text { System - SICA }\end{array}$ & $\begin{array}{l}\text { Regional Program for Food and Nutrition } \\
\text { Security for Central America } \\
\text { (PRESANCA II) }\end{array}$ & programa \\
\hline
\end{tabular}

Fuente: elaboración propia sobre la base de SEGIB 2018b, pp. 147-149.

ración económica en la primera década del siglo XXI, se abrieron o fortalecieron varios espacios regionales, así como también se crearon nuevas condiciones para el debate sobre la cooperación a nivel nacional. Un ejemplo de este tipo de "espacios regionales" en los que la cooperación entre los Estados miembros desempeña un papel singular son ALBA, UNASUR o CELAC (SEGIB 2018a: 58-59). También se debe enfatizar el potencial creciente de este tipo de cooperación, como se evidencia al incluirla en las estadísticas mantenidas por SEGIB en lugar de los datos sobre la AOD. A partir de 2013, los informes de SEGIB sobre la cooperación Sur-Sur en América Latina incluyen datos sobre las iniciativas implementadas bajo la cooperación regional Sur-Sur en lugar de datos referentes a la AOD.

\section{Conclusiones}

La gobernanza de la seguridad alimentaria es un proceso complejo que requiere la consideración de muchos factores. Debido a su naturaleza integral, requiere acciones coordinadas tomadas en varios niveles organizacionales. 
Además, reconocer la lucha contra el hambre y la desnutrición como un problema prioritario dentro del marco de la Agenda 2030 impone obligaciones especiales a los Estados en esta área.

Nuestro análisis indica que en América Latina se ha conseguido desarrollar un modelo regional de la gobernanza de la seguridad alimentaria, basado en la cooperación bilateral y multilateral de los países de la región dentro del marco de la así llamada cooperación Sur-Sur. Este modelo está en línea con la filosofía de la Agenda 2030, así como la especificidad de la seguridad alimentaria condicionada por el contexto regional, ya que son otros factores que afectan el nivel de la seguridad alimentaria en África, otros en Asia y otros en América Latina. El análisis realizado permite formular tres conclusiones. Primero, frente a los nuevos compromisos en la lucha contra el hambre y la desnutrición (ODS-2), así como a los flujos decrecientes de la Ayuda Oficial al Desarrollo en el marco de la cooperación Norte-Sur, los países en desarrollo deben buscar nuevas soluciones y formas de cooperación. En segundo lugar, la cooperación intrarregional en la fórmula Sur-Sur se basa en una mejor adaptación de la oferta de asistencia a las necesidades de los países beneficiarios y una mayor participación de los donantes, ya que ambas funciones son desempeñadas simultáneamente por los países involucrados. En tercer lugar, la politización y la regionalización de la lucha contra el hambre y la desnutrición en América Latina, como lo demuestran las iniciativas de cooperación Sur-Sur implementadas, contribuyen a cambiar la percepción de los problemas de seguridad alimentaria y adoptar un enfoque más integral, el cual tiene en cuenta las actividades no solo en el campo de la agricultura, sino también en el ámbito de la protección de la salud, la lucha contra el cambio climático o la reforma de la administración pública.

\section{Referencias bibliográficas}

Abdenur Erthal A., Da Fonseca Marques J.M.E. (2013), The North's Growing role in South-South Cooperation: Keeping the Foothold, “Third World Quarterly", vol. 34, no 8, pp. 1475-1491.

BAPA (1978), Buenos Aires Action Plan of Action for Promoting and Implementing Technical Cooperation among Developing Countries, The United Nations Conference on Technical Cooperation among Developing Countries, Buenos Aires 30 August - 12 September 1978, https://drive.google.com/file/d/0B-buqyonV0jpSMm1OVEZYU2hNTWc/view, fecha de consulta: 23.04.2019.

Candel J.J.L. (2014), Food Security Governance: A Systematic Literature Review, "Food Security", vol. 6, pp. 585-601.

CEPAL (2018), Social Panorama of Latin America 2018, https://repositorio.cepal.org/ bitstream/handle/11362/44396/4/S1900050_en.pdf, fecha de consulta: 2.05.2019.

CEPAL, FAO, ALADI (2016), Food and Nutrition Security and the Eradication of Hunger CELAC 2025. Furthering Discussion and Regional Cooperation, https://repositorio.cepal. org/handle/11362/40355, fecha de consulta: 2.05.2019.
La gobernanza de la seguridad alimentaria en América Latina: desde la cooperación Norte-Sur hacia la cooperación Sur-Sur

Katarzyna Marzęda-Młynarska 
Dossier América Latina: continuidad y cambio en el escenario regional
FAO (2009), Declaration of the World Summit on Food Security, WSFS, Rome 16-18 November 2009.

FAO (2018), The State of Food Security and Nutrition in the World 2018, http://www.fao. org/3/I9553EN/i9553en.pdf, fecha de consulta: 23.04.2019.

FAO (1996), Rome Declaration on World Food Security and World Food Summit Plan of Action, World Food Summit, Rome 13-17 November 1996.

FAO (2011), Good Food Security Governance: The Crucial Premise to the Twin-Track Approach, Background paper, ESA Workshop, Rome 5-7 December 2011.

FAO (2017), Climate Change and Food Security and Nutrition in Latin America and the Caribbean, Santiago.

FAO, PAHO, WHO (2017), Panorama of Food and Nutrition Security in Latin America and Caribbean, Santiago de Chile, http://www.fao.org/3/a-i7914e.pdf, fecha de consulta: 2.05.2019.

GFSI (2018), Global Food Security Index 2018. Building Resilience in the Face of Rising Food Security Risks, "The Economist" Intelligence Unit, https://foodsecurityindex.eiu.com/, fecha de consulta: 23.04.2019.

Gómez Ramírez E. (2019), South-South and Triangular Cooperation in Latin America, European Parliamentary Research Service, PE 635.607, March 2019, http://www.europarl. europa.eu/RegData/etudes/BRIE/2019/635607/EPRS_BRI(2019)635607_EN.pdf, fecha de consulta: 23.04.2019.

IFPRI (2018), Climate Change and Agriculture in Central America and the Andean Region, http://ebrary.ifpri.org/utils/getfile/collection/p15738coll2/id/132953/filename/133157.pdf, fecha de consulta: 24.04.2019.

Koba M. (2014), Why the Next World War Will Be Fought Over Food, "Fortune", December 21, URL: http://fortune.com/2014/12/21/why-the-next-world-war-will-be-fought-overfood/, fecha de consulta: 12.03.2019.

Marzęda-Młynarska K. (2014), Globalne zarządzanie bezpieczeństwem żywnościowym na przełomie XX i XXI wieku, Wydawnictwo UMCS, Lublin.

UN (2015a), Transforming Our World: the 2030 Agenda for Sustainable Development, Resolution adopted by the General Assembly on 25 September 2015, A/Res/70/1.

UN (2015b), Addis Ababa Action Agenda of the Third International Conference on Financing for Development, Addis Ababa, Ethiopia 13-16 July 2015, GA Resolution 69/313 of 27 July 2015.

UN (2015c), The Millennium Development Goals Report 2015, https://www.un.org/ millenniumgoals/2015_MDG_Report/pdf/MDG\%202015\%20rev\%20(July\%201).pdf, fecha de consulta: 23.04.2019.

UN (2017), Sustainable Development Goals Report 2017, https://unstats.un.org/sdgs/files/report/2017/TheSustainableDevelopmentGoalsReport2017.pdf, fecha de consulta: 23.04.2019.

UN (2018), Sustainable Development Goals Report 2018, https://unstats.un.org/sdgs/ report/2018, fecha de consulta: 23.04.2019.

UN (2019), Buenos Aires Outcome Document of the Second High-Level United Nations Conference on South-South Cooperation, Draft resolution submitted by the President of the General Assembly, A/73/L.80, https://undocs.org/en/A/73/L.80, fecha de consulta: 23.04.2019. 
Pèrez-Escamilla R., Shamah-Levy T., Candel J. (2017), Food Security Governance in Latin America: Principles and Way Forward, "Global Food Security", no 14, pp. 68-72.

Piñeiro M., Bianchi E., Uzquiza L., Trucco M. (2010), Food Security Policies in Latin America, Series on Trade and Food Security - Policy Report 4, International Institute for Sustainable Development.

SEGIB (2018a), A Decade of South-South Cooperation in Ibero-America 2007/2017, https:// www.informesursur.org/?page_id=4723\&lang=en, fecha de consulta: 16.05.2019.

SEGIB (2018b), Report on South-South Cooperation in Ibero-America 2018, Ibero-American General Secretariat, https://www.segib.org/en/?document=informe-de-cooperacion-sursur-en-iberoamerica-2018, fecha de consulta: 23.04.2019.

Shaw D.J. (2007), World Food Security. A History Since 1945, Basingstoke - New York.

UNOSSC (2019), About South-South and Triangular Cooperation, UNOSSC official website, https://www.unsouthsouth.org/about/about-sstc/, fecha de consulta: 23.04.2019.
La gobernanza de la seguridad alimentaria en América Latina: desde la cooperación Norte-Sur hacia la cooperación Sur-Sur

Katarzyna Marzęda-Młynarska 